\title{
Uma Revisão Sistemática da Literatura de Jogos Digitais Voltados para o TDAH
}

\author{
Vinícius D. J. Maciel $^{1}$, Bruno C. Matias $^{1}$, Victor T. Sarinho ${ }^{1}$ \\ ${ }^{1}$ Laboratório de Entretenimento Digital Aplicado - LEnDA \\ Universidade Estadual de Feira de Santana - UEFS \\ Feira de Santana - Bahia - Brasil \\ viniciusmacieluefs@outlook.com, brunoclaudinomatias@gmail.com, \\ vsarinhoduefs.br
}

\begin{abstract}
People affected by Attention Deficit Hyperactivity Disorder (ADHD) tend to present greater difficulties in routine activities than people who do not have the disorder. These effects, which begin in childhood, can continue throughout life. This article presents a literature review of developed digital games and tools aimed at reducing the impact of these symptoms on the life of those who have the disorder. It is a survey that show the current research trends in the area, as well as possible lines of research not explored in the context of digital games applied to ADHD.
\end{abstract}

Resumo. Pessoas afetadas pelo Transtorno de Déficit de Atenção e Hiperatividade (TDAH) tendem a apresentar maiores dificuldades em atividades rotineiras que pessoas que não possuem o transtorno. Esses efeitos, que começam na infância, podem continuar por toda a vida. Este artigo apresenta uma revisão da literatura de jogos e ferramentas digitais desenvolvidas com o intuito de reduzir o impacto desses sintomas na vida de quem possui o transtorno. Trata-se de um levantamento que busca mostrar as tendências atuais de pesquisa na área, bem como possíveis linhas de pesquisa não exploradas no contexto de jogos digitais aplicados ao TDAH.

\section{Introdução}

O Transtorno de Déficit de Atenção e Hiperatividade (TDAH) é considerado um dos distúrbios neuropsiquiátricos mais comum na infância [Fontana et al. 2007], [Siqueira and Gurgel-Giannetti 2011]. Ele traz como principais características a desatenção, a hiperatividade e a impulsividade [Catelan-Mainardes 2010], podendo afetar o desempenho do indivíduo no âmbito social, profissional e escolar como um todo [Rohde et al. 2004].

Apesar do distúrbio geralmente se iniciar antes dos sete anos de idade, este é melhor diagnosticado quando a criança ingressa na escola. Isso se deve ao fato de ser o período em que as dificuldades de atenção e inquietude são percebidas com maior frequência, quando comparadas com outras crianças de mesma idade [Couto et al. 2010]. Como resultado, o TDAH representa, junto com a dislexia, a principal causa de fracasso escolar de crianças e adolescentes em geral [Couto et al. 2010].

Jogos digitais tem se tornado a terceira atividade mais comum entre os usuários de computadores e smartphones do Brasil [Rocha et al. 2014]. Mais ainda, o número 
de jovens e crianças que escolhem os jogos digitais como a principal atividade em suas horas livres tem crescido de forma constante nos últimos anos em todo o mundo [Rocha et al. 2014]. Além disso, autores como [Kirby 1995], aconselha o uso de jogos para aplicar princípios de aprendizado ativo, usando-os para garantir que os participantes aprendam melhor.

O jogo proporciona aprendizado por descobrimento, permitindo-os fazer, ler, ouvir e observar em conjunto o todo [Peletti 2015]. Assim, jogos digitais podem se tornar uma ótima maneira de melhorar o contato com indivíduos, tendo como resultado um espaço significativo para a aplicação dos mesmos no âmbito da reabilitação de jovens e adultos em geral [Rocha et al. 2014].

Diferentes jogos digitais tem sido desenvolvidos e utilizados nos últimos anos para o tratamento e o apoio aos portadores de TDAH. Este artigo apresenta uma revisão sistemática da literatura de jogos digitais voltados para o TDAH, descrevendo as linhas de trabalho conduzidas por tais jogos, bem como identificando possíveis áreas de pesquisa futuras relacionadas ao tema em si.

\section{Metodologia}

\subsection{Critérios de Inclusão e Exclusão}

Para a elaboração desta revisão sistemática de jogos digitais voltados para o TDAH, utilizou-se a plataforma Google Scholar, onde foram filtrados artigos científicos relacionados as seguintes palavras chaves: "Jogos para TDAH", "Games for ADHD", "TDAH", "Ferramental digital para TDAH", "Diagnóstico TDAH", entre outros. Foram considerados apenas artigos publicados nos últimos anos em que a redação estivesse em um dos idiomas filtrados, neste caso inglês ou português.

Além disso, para uma revisão sistemática da literatura, é necessário uma sistematização de perguntas as quais servirão para validar publicações relevantes. As questões que foram usadas como guia para este estudo foram:

- QP1. O estudo construiu algum jogo ou dinâmica de jogo para avaliação, tratamento, conscientização e/ou demais ações relacionadas ao TDAH?

- QP2. O estudo apresenta algum modelo de interface ou abordagem de interação diferenciada capaz de ser aplicada em jogos ou dinâmicas de jogos para pessoas que sofrem TDAH?

- QP3. O estudo apresenta alguma característica particular de design de jogos ou estratégia de produção de jogos voltada para pessoas que sofrem TDAH?

Os artigos aprovados pelas questões de pesquisa ainda precisam passar por uma avaliação de qualidade dos mesmo, onde são feitas as seguintes perguntas sobre o seu conteúdo:

- Q1. O estudo desenvolveu algum jogo, projeto de jogo ou dinâmica de jogo?

- Q2. O estudo apresenta o que é necessário para se criar um jogo voltado para pessoas portadoras de TDAH?

- Q3. O estudo apresenta algum método novo para avaliação, tratamento, conscientização e/ou demais ações relacionadas ao TDAH?

- Q4. O estudo apresenta alguma verificação, validação ou abordagem de teste dos resultados apresentados? 
- Q5. O estudo apresenta resultados positivos nas suas conclusões relacionadas a sua aplicação no TDAH?

Cada questão foi respondida com $\operatorname{Sim}(\mathrm{S}), \operatorname{Parcial}(\mathrm{P})$ ou Não(N) com as notas 1 , 0.5 ou zero pontos, respectivamente. Dessa forma, os melhores artigos seriam aqueles em que a nota mais se aproxima de 5.

\subsection{Resultado da Pesquisa}

A $1^{a}$ fase de pesquisa compreende a busca conforme critérios de inclusão e exclusão definidos anteriormente. $\mathrm{Na} 2^{\mathrm{a}}$ fase, os estudos selecionados pela $1^{\mathrm{a}}$ fase passam por um processo tentando indentificar no título e resumo os termos da pesquisa. E na $3^{\text {a }}$ fase, são considerados apenas os estudos em que há alguma contribuição direta com jogos para TDAH conforme as questões QP1, QP2 e QP3 de validação dos artigos. Vale salientar que o tempo total utilizado para pesquisa, filtragem, revisão e avaliação dos artigos foi de aproximadamente 30 dias.

Dessa forma, na Tabela 1, é apresentado o total de artigos analisados no decorrer de cada fase. Já a Tabela 2 , esta informa os resultados obtidos após a filtragem na $3^{\text {a }}$ fase, onde foram selecionados 9 artigos referentes a jogos digitais e TDAH a serem analisados.

Tabela 1. Quantidade de artigos encontrados por fase.

\begin{tabular}{|c|c|c|}
\hline $1^{\mathrm{a}}$ Fase & $2^{\mathrm{a}}$ Fase & $3^{\mathrm{a}}$ Fase \\
\hline 4280 & 24 & 9 \\
\hline
\end{tabular}

Tabela 2: Estudos selecionados na $3^{\text {a }}$ fase.

\begin{tabular}{|l|l|l|l|l|}
\hline Artigo & Ano & \multicolumn{1}{|c|}{ Título } & Citações \\
\hline A1 & 2013 & $\begin{array}{l}\text { Jogos Computacionais e Transtorno de Déficit de Atenção e Hipera- } \\
\text { tividade: Revisão Sistemática de Literatura [Oliveira et al. 2013] }\end{array}$ & 8 \\
\hline A2 & 2001 & $\begin{array}{l}\text { Trasntorno do déficit de atenção e hiperatividade Proposta de } \\
\text { avaliação neuropsicológica para diagnóstico [Amaral et al. 2001] }\end{array}$ & 63 \\
\hline A3 & 2016 & $\begin{array}{l}\text { Computer Game To Identify Characteristics Of ADHD } \\
\text { [Silva et al. 2016] }\end{array}$ & - \\
\hline A4 & 2010 & $\begin{array}{l}\text { Use of digital console game for children with attention deficit hype- } \\
\text { ractivity disorder [Chuang et al. 2010] }\end{array}$ & 5 \\
\hline A5 & 2006 & $\begin{array}{l}\text { Supermarket Game: An Adaptive Intelligent Computer } \\
\text { Game for Attention Deficit/Hyperactivity Disorder Diagnosis } \\
\text { [De Andrade et al. 2006] }\end{array}$ & 12 \\
\hline A6 & 2016 & $\begin{array}{l}\text { Gamebook e a estibulação de funções executivas em crianças com } \\
\text { indicação de diagnóstico de TDAH [Alves and Bonfim 2016] }\end{array}$ & 5 \\
\hline A7 & 2015 & $\begin{array}{l}\text { An iPad-Based Tool for Improving the Skills of Children with Atten- } \\
\text { tion Deficit Disorder [Wrońska et al. 2015] }\end{array}$ & 16 \\
\hline A8 & 2010 & $\begin{array}{l}\text { Utilização de Jogos Virtuais na Prática Educacional de Crianças } \\
\text { com Transtorno de Déficit de Atenção e/ou Hiperatividade } \\
\text { [Guimarães and Ribeiro 2010] }\end{array}$ & 2 \\
\hline A9 & 2007 & $\begin{array}{l}\text { Ambientes Virtuais na prática educacional de crianças com Trans- } \\
\text { torno de déficit de atenção ou hiperatividade [Guimarães et al. 2007] }\end{array}$ & 3 \\
\hline
\end{tabular}


Os artigos selecionados na $3^{\text {a }}$ fase, como citado anteriormente, passam para a próxima etapa da revisão que é a avaliação de qualidade dos artigos selecionado. Para realizar isso, os artigos foram lidos e analisados pelas questões de qualidade apresentadas na seção anterior (Q1, Q2, Q3, Q4 e Q5). A avaliação de cada estudo é apresentada na Tabela 3, com suas respectivas pontuações. Vale salientar que os artigos com pontuação abaixo de 1 se devem ao fato de ou ser uma revisão da literatura ou não se adequar a nenhuma das questões de qualidade propostas, mesmo possuindo características que o fizeram ser escolhidos pela $3^{\text {a }}$ fase.

Tabela 3. Avaliação de qualidade

\begin{tabular}{|c|c|c|c|c|c|c|}
\hline Artigo & Q1 & Q2 & Q3 & Q4 & Q5 & Pontos \\
\hline A1 & N & N & N & N & N & 0 \\
\hline A2 & N & N & P & N & N & 0.5 \\
\hline A3 & S & S & P & S & S & 4.5 \\
\hline A4 & S & N & P & S & S & 3.5 \\
\hline A5 & S & N & P & N & N & 1.5 \\
\hline A6 & S & S & S & S & S & 5.0 \\
\hline A7 & S & S & S & S & S & 5.0 \\
\hline A8 & S & S & P & S & S & 4.5 \\
\hline A9 & S & S & P & S & S & 4.5 \\
\hline
\end{tabular}

\section{Resultados \& Discussões}

Para organizar a discussão sobre os artigos selecionados, foi considerado primeiramente os artigos voltados para o diagnóstico do TDAH, pois esses foram encontrados em menor quantidade e possuem objetivos fixos e técnicas mais detalhadas. Logo em seguida são apresentados os artigos voltados ao tratamento do transtorno com jogos digitais. Dentro de cada subtópico, a ordem utilizada para discutir sobre os artigos foi a ordem de seleção da tabela. Entretanto, o primeiro trabalho [Oliveira et al. 2013], não é discutido neste tópico, pois o mesmo, apesar de passar nas medidas de qualidade para artigos, não apresenta jogo ou ferramenta digital que possa ser discutida.

\subsection{Jogos de Apoio ao Diagnóstico de TDAH}

O diagnóstico do TDAH é clínico, onde o médico procura observar o comportamento social da criança [Couto et al. 2010]. Apesar de não existir uma bateria fixa de testes para avaliar as crianças com TDAH [Amaral et al. 2001], é possível realizar uma proposta mais simples de avaliação que aplica vários tipos de testes já existentes, a exemplo do Wisconsin Card Sorting Test (WCST) [Amaral et al. 2001] que possui diferentes adaptações disponíveis na web conforme ilustrado na Figura 1. Trata-se de um instrumento desenvolvido para avaliar a capacidade do indivíduo de raciocinar de forma abstrata, e de modificar suas estratégias cognitivas como resposta a alterações nas contingências ambientais [Miguel 2005].

Além do WCST, há outro estudo, em que o uso de cores foi aplicado para o diagnóstico de TDAH através de jogos digitais, uma vez que pessoas com TDAH, apresentam um prejuízo na percepção de algumas cores do eixo azul-amarelo [Silva et al. 2016]. 


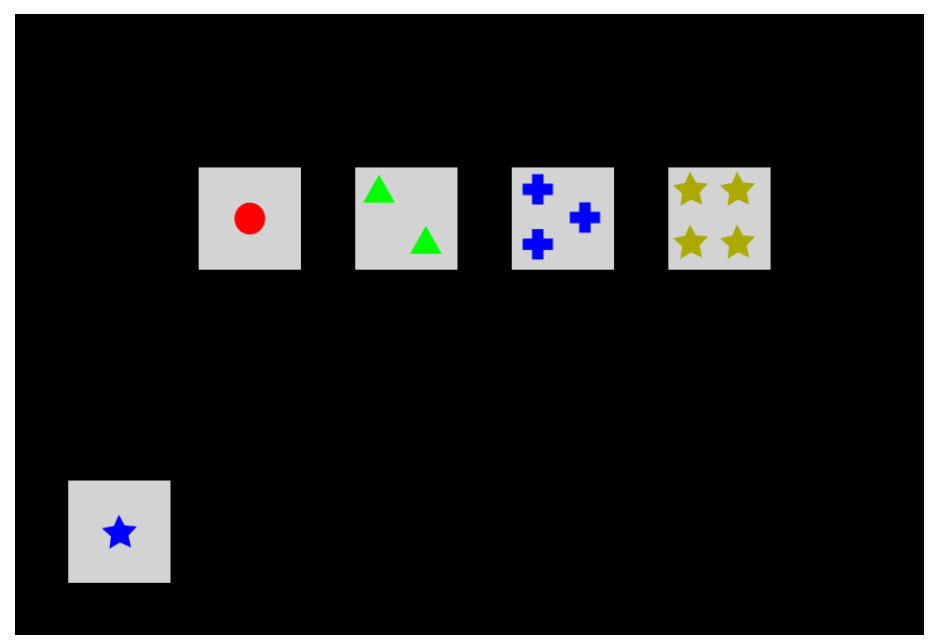

Figura 1. Versão Web do WCST. Imagem retirada do site disponível online.

Para a avaliação desse estudo [Silva et al. 2016], foi proposto um jogo composto de duas fases de aventura, com ambientes diferentes e cores predominantes do eixo azulamarelo (Figura 2). Nesse jogo não há punição de erros, mas sim alertas sobre os mesmos cometidos, com o devido auxílio sonoro [Silva et al. 2016]. Registros quantitativos do desempenho de jogadores, em relação aos critérios de diagnóstico descritos pelo Continuous Performance Test III, também são fornecidos pelo jogo, possibilitando assim caracterizar sintomas de TDAH nos respectivos jogadores.
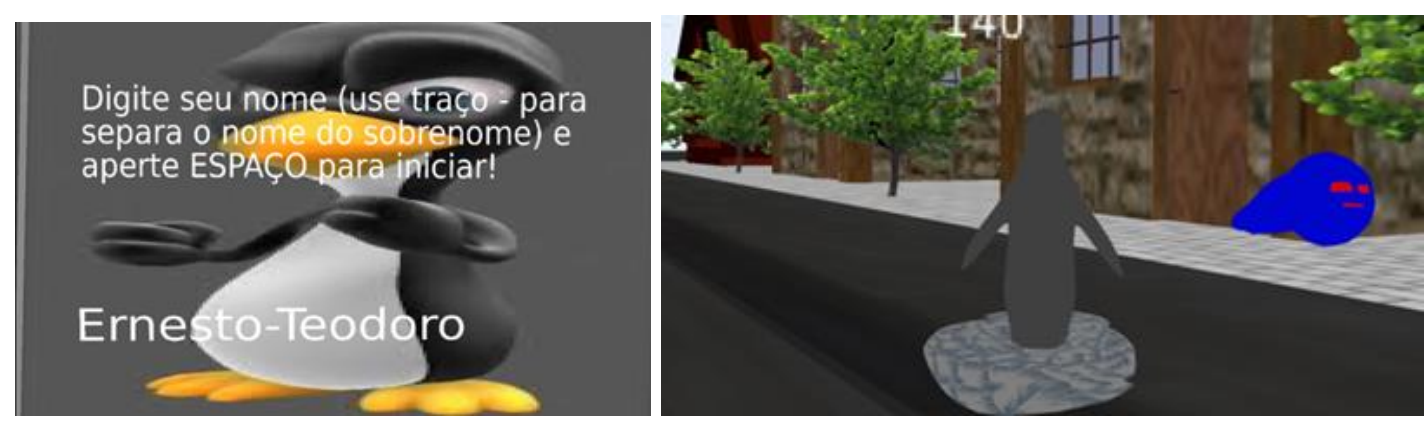

Figura 2. Jogo baseado no uso de cores para o diagnóstico de TDAH. Imagem retirada do artigo original [Silva et al. 2016].

\subsection{Jogos de Apoio ao Tratamento de TDAH}

Com relação ao uso de jogos como ferramenta de apoio ao tratamento de TDAH, identificou-se um estudo que combina elementos de terapia comportamental e de teoria do comportamento cognitivo com elementos e conceitos de jogos digitais, de modo a desenvolver um tratamento para melhorar a atenção seletiva de pessoas portadoras do transtorno [Chuang et al. 2010]. Nele, participantes foram divididos em dois grupos, sendo um grupo tratado com terapia de comportamento cognitivo normal, e o outro sendo tratado com o apoio de jogos do Nintendo Wii, tais como "Shoting Range", "Find Mii" (Figura 3), "Table Tennis" e "Wii Sport" [Chuang et al. 2010]. Como resultado, foi percebido que o uso desses jogos tinham um grande impacto na motivação e na concentração das crianças enquanto estavam sendo tratadas [Chuang et al. 2010]. 


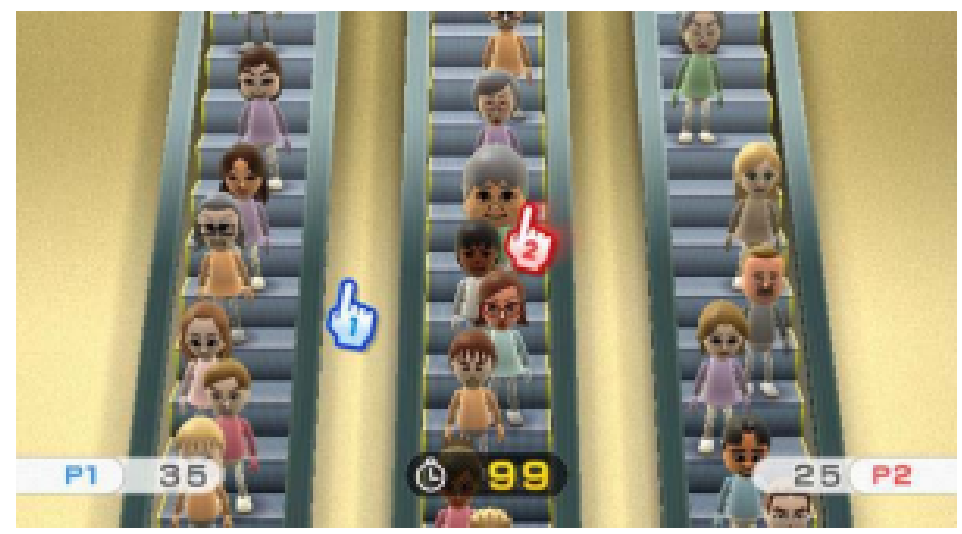

Figura 3. Jogo Find Mii utilizado como ferramenta de apoio ao TDAH. Imagem encontrada em [Chuang et al. 2010].

Outro exemplo de jogo de apoio ao tratamento de TDAH é o The Supermarket Game [De Andrade et al. 2006]. Trata-se de um jogo de labirinto em que o personagem precisa pegar os itens da lista de compras que estão nos corredores do supermercado sem passar duas vezes pelo mesmo caminho (Figura 4). O jogo utiliza muito a mecânica de estimular a capacidade de planejamento, já que é necessário pensar no caminho a ser percorrido antes de iniciar o trajeto [De Andrade et al. 2006]. Além disso, o jogo procura também estimular o controle inibitório, realizando descontos na pontuação caso as regras sejam quebradas [De Andrade et al. 2006].

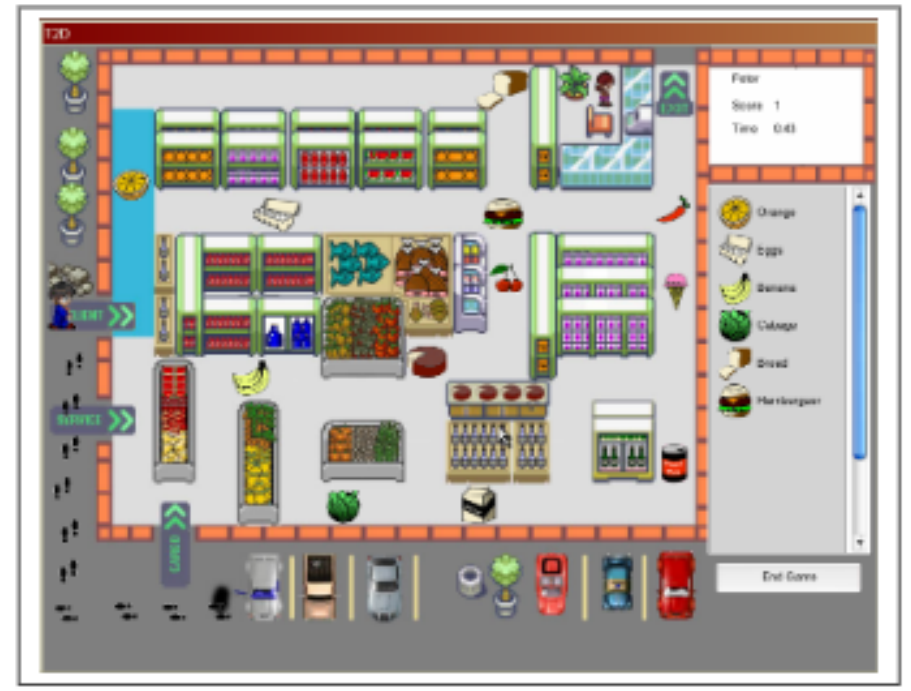

\section{Figura 4. Tela principal do The Supermarket Game. Figura retirada de [De Andrade et al. 2006].}

Outra trabalho desenvolvido na área de tratamento do TDAH é o Gamebook (Figura 5), trata-se de uma mídia híbrida com características de jogo digital e appbook, que foi desenvolvida para estimular as funções executivas de crianças na faixa etária de 8 a 12 anos, em especial para aquelas com diagnóstico de TDAH [Alves and Bonfim 2016]. Entre as histórias que o jogo possui, a criança passa por 8 mini-jogos, os quais tem como objetivo principal potencializar as funções executivas de memória de trabalho, planeja- 
mento, flexibilidade cognitiva, categorização, controle inibitório e atenção seletiva do jogador [Alves and Bonfim 2016].

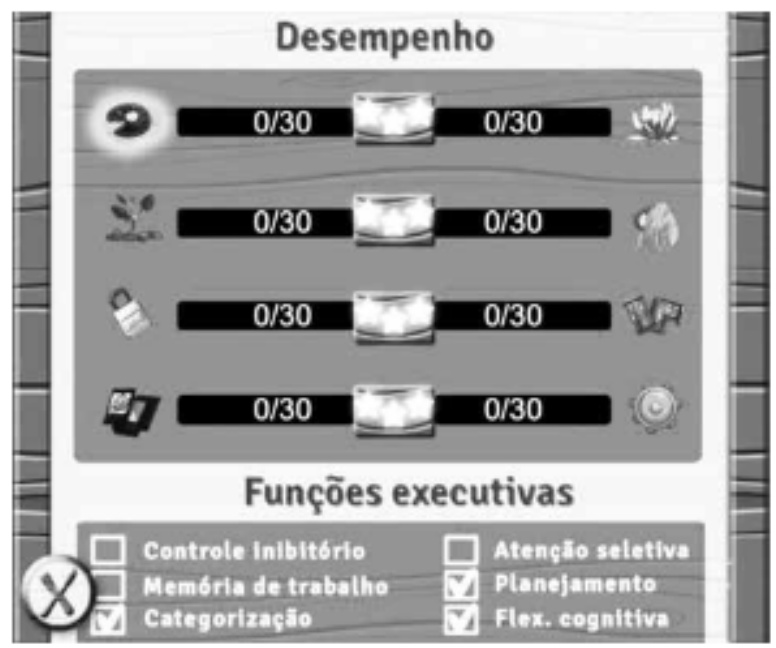

Figura 5. Tela de resultados de desempenho do jogador no Gamebook. Imagem obtida do artigo original [Alves and Bonfim 2016].

Além desses é citado em [Wrońska et al. 2015] uma plataforma desenvolvida para iPad em formato de jogo, que tem como foco avaliar a qualidade de leitura das crianças que possuem TDAH, por meio da interação do público infantil com o iPad. O jogo proposto, consiste em o paciente ler uma pequena frase que especifica uma instrução, depois entender o que foi lido, selecionar um dos botões da barra de ferramentas na lateral direita (Figura 6), e por fim arrastar esse botão selecionado até a imagem ou o rotúlo predeterminado [Wrońska et al. 2015]. Como resultado, percebeu-se num grande aumento tanto na atenção das crianças, como também em sua compreensão textual [Wrońska et al. 2015].

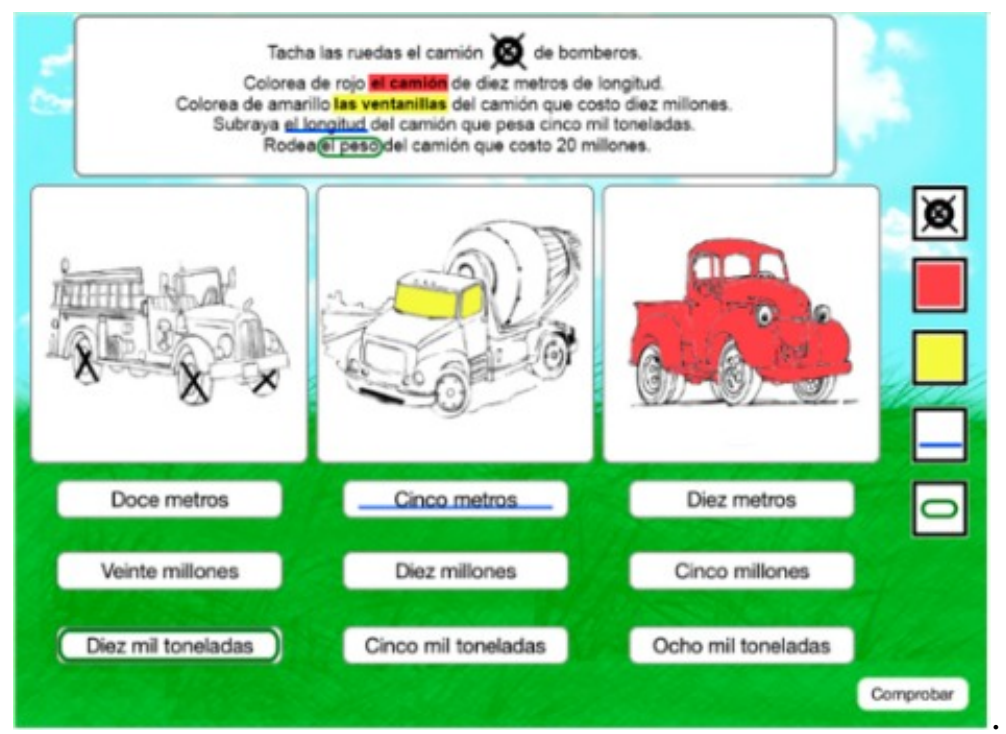

Figura 6. Tela de uma das atividades do jogo desenvolvido para iPad. Imagem obtida do artigo [Wrońska et al. 2015]

Já em [Guimarães and Ribeiro 2010] são apresentados 3 tipos diferentes de jogos 
desenvolvidos no ambiente Island Demo que buscam uma melhora no aprendizado de crianças com TDAH. Estes jogos são:

- "Math" (Figura 7(a)), que por meio de figuras geométricas com pontuações individuais agregadas a cada uma das figuras, visa trabalhar com a resolução de exercícios matemáticos simples, estimulando o desenvolvimento da atenção com níveis crescentes de dificuldade [Guimarães and Ribeiro 2010];

- "Cores e Formas" (Figura 7(b)), que tem como finalidade trabalhar a memorização de sequência de formas geométricas e trabalhar a atenção, concentração e o raciocinio lógico da criança; e

- "Caça ao tesouro" (Figura 7(c)), que tem como objetivo trabalhar a cordenação motora do jogador, além de beneficiar também a concentração e a persistência nas tarefas [Guimarães and Ribeiro 2010].

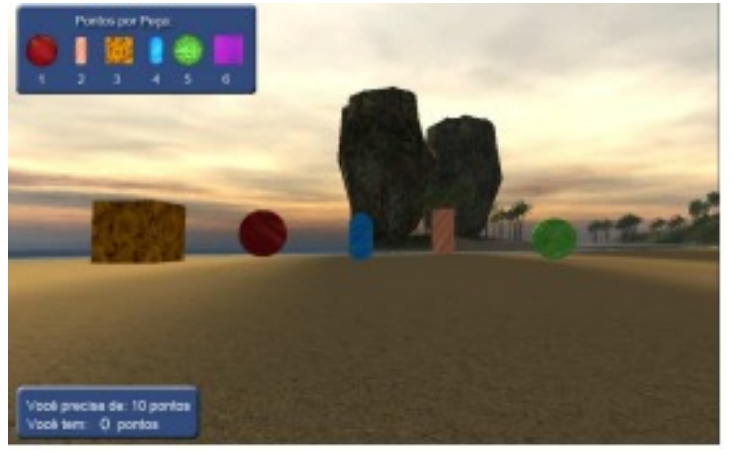

(a) Math

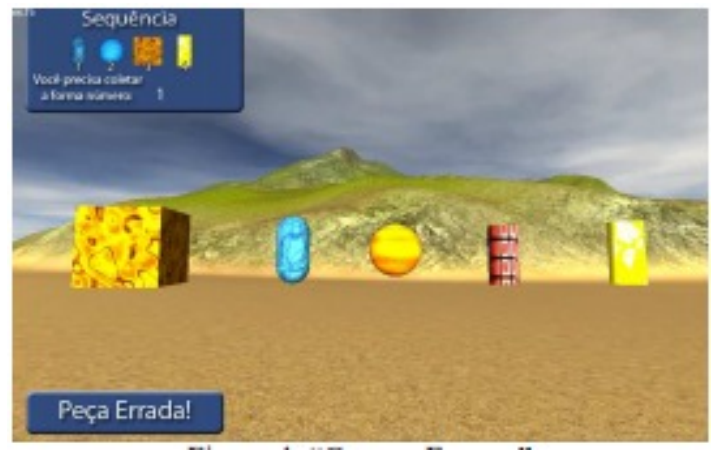

(b) Cores e Formas

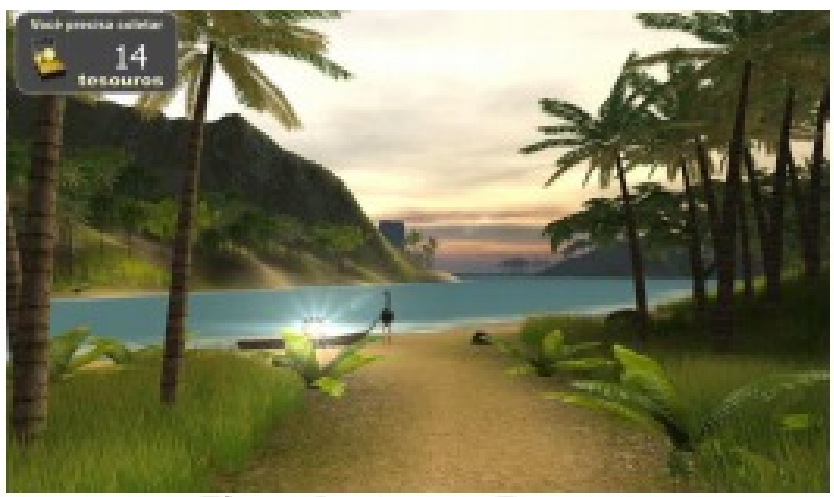

(c) Caça ao Tesouro

Figura 7. Jogos digitais desenvolvidos no ambiente Island Demo.

Para finalizar, MemoZoo (Figura 8) representa um ambiente virtual 3D em formato de jogo que mobiliza estruturas cognitivas simples e busca obter uma maior rentabilidade escolar da criança com TDAH [Guimarães et al. 2007]. Trata-se de uma plataforma onde são apresentados exercícios de matemática básica de múltipla escolha, em conjunto com caixas virtuais de onde surgem animais capazes de formar pares como em um jogo da memória [Guimarães et al. 2007].

\section{Conclusões e Trabalhos Futuros}

Jogos sérios possuem finalidades que vão além do entretenimento, usando a tecnologia a favor do aprendizado [Oliveira et al. 2013]. Eles são bons mediadores capazes de pro- 


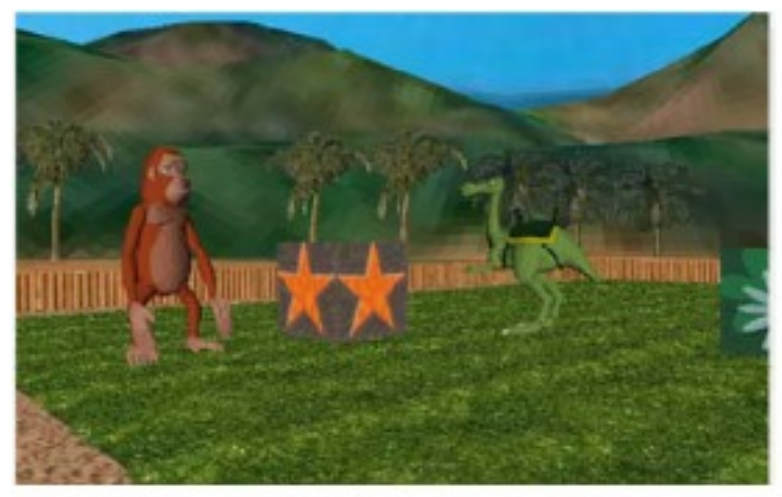

Figura 8. Cenário 3D do jogo MemoZoo. Imagem retirada de [Guimarães et al. 2007].

porcionar ao jogador imersão, atenção, conhecimento funcional, definição e objetivos, autocontrole, tomada de decisão, auto-eficácia e motivação interna [Oliveira et al. 2013].

Jogos digitais também podem ser de grande ajuda para o tratamento e o apoio de pessoas portadoras do TDAH, algo que pode ser confirmado a partir dos trabalhos descritos que demonstram um certo otimismo sob a perspectiva de uma melhora no tratamento e suporte de pessoas portadoras do TDAH. Um bom exemplo desta afirmação pode ser confirmada pelo experimento MemoZoo [Guimarães et al. 2007] com grupos de crianças portadoras de TDAH ou não, as quais apresentaram um grande aumento no interesse pelo jogo no ambiente de Realidade Virtual, mostrando um grande potencial e diferencial na área na retenção da atenção e no maior engajamento do público infantil do que outras mídias tradicionais. Já no experimento do Gamebook [Alves and Bonfim 2016], há uma vasta consultoria da opinião de diferentes psicólogos para diferentes áreas de estímulos explorados dentro dos jogos propostos como: Memória de Trabalho, Planejamento, Controle Inibitório, Atenção Seletiva, Categorização e Flexibilidade Cognitiva, confirmando o potencial dos jogos digitais no tratamento e suporte de pessoas portadoras do TDAH.

Porém em outros trabalhos, os resultados na parte prática não foram tão rentáveis, tendo uma carência muito grande de uma amostragem mais técnica com participação de profissionais da área, ou até mesmo de um experimento com diferentes grupos de crianças que teriam ou não TDAH. Mesmo que houvesse um projeto bem consolidado, com um software em pleno funcionamento, é necessária uma amostragem que represente a efetividade do uso de jogos digitais nos diferentes grupos infantis.

Contudo, apesar da dificuldade de se encontrar jogos digitais de qualidade para o TDAH, houve também uma carência de especificações dos resultados obtidos em alguns projetos, principalmente quando se questionava o tempo e o interesse que cada criança diagnosticada com TDAH tinha pelo jogo proposto. Também não foram encontrados jogos ou ferramentas digitais voltados para a conscientização do TDAH, algo que pode sinalizar a atual ausência de campanhas educacionais e de promoção a saúde relacionadas ao respectivo tema.

Como trabalhos futuros, é previsto a criação de jogos sérios voltados para cobrir a atual carência de soluções de apoio ao diagnóstico de TDAH. Para tal, serão analisadas diferentes possibilidades de gamificação para as abordagens atuais disponíveis 
de diagnóstico do TDAH. Trabalhos que apliquem o entretenimento computacional na conscientização da população sobre os problemas que o TDAH causa na vida de um indivíduo, portador ou não do transtorno, também serão desenvolvidos em um futuro próximo. Almeja-se uma maior qualidade nos softwares desenvolvidos para esta área, visando atender com eficiência o público infantil com TDAH, além de melhorar o acesso ao grande público a informação sobre o Transtorno e de como pode ser amenizado e tratado com a maior qualidade possível.

\section{Referências}

Alves, L. and Bonfim, C. (2016). Gamebook e a estimulação de funções executivas em crianças com indicação de diagnóstico de tdah: Processo de pré-produção, produção e avaliação do software. Revista da FAEEBA-Educação e Contemporaneidade, 25(46).

Amaral, A. H. d., Guerreiro, M. M., et al. (2001). Transtorno do déficit de atenção e hiperatividade: proposta de avaliação neuropsicológica para diagnóstico. Arquivos de Neuro-Psiquiatria.

Catelan-Mainardes, S. C. (2010). Transtorno de déficit de atenção e hiperatividade na infância e adolescência pela perspectiva da neurobiologia. Saúde e Pesquisa, 3(3).

Chuang, T.-Y., Lee, I., et al. (2010). Use of digital console game for children with attention deficit hyperactivity disorder. Online Submission, 7(11):99-105.

Couto, T. S., Melo-Junior, M. R., and Gomes, C. R. A. (2010). Aspectos neurobiológicos do transtorno do déficit de atenção e hiperatividade (tdah): uma revisão. Ciências \& Cognição, 15(1):pp-241.

De Andrade, L. C. V., Carvalho, L. A. V., Lima, C., Cruz, A., Mattos, P., Franco, C., Soares, A., and Grieco, B. (2006). Supermarket game: an adaptive intelligent computer game for attention deficit/hyperactivity disorder diagnosis. In Artificial Intelligence, 2006. MICAI'06. Fifth Mexican International Conference on, pages 359-368. IEEE.

Fontana, R. d. S., Vasconcelos, M. M. d., Werner Júnior, J., Góes, F. V. d., and Liberal, E. F. (2007). Prevalência de tdah em quatro escolas públicas brasileiras. Arq neuropsiquiatr, pages 134-137.

Guimarães, M., Carvalho, L., and Costa, R. (2007). Ambientes virtuais na prática educacional de crianças com transtorno de déficit de atenção e/ou hiperatividade. In SYMPOSIUM ON VIRTUAL AND AUGMENTED REALITY, volume 9.

Guimarães, M. d. S. and Ribeiro, P. C. (2010). Utilização de jogos virtuais na prática educacional de crianças com transtorno de déficit de atenção e/ou hiperatividade. IX SBGames.

Kirby, A. (1995). 150 Jogos de treinamento.

Miguel, F. K. (2005). Teste Wisconsin de Classifica $\tilde{\AA} \S \tilde{A} d e$ Cartas. Avalia $\tilde{A} \S \tilde{A P s i c o l \tilde{A}}$, $4: 203-204$.

Oliveira, L. B., Ishitani, L., and Cardoso, A. M. (2013). Jogos computacionais e transtorno de déficit de atenção e hiperatividade: Revisão sistemática de literatura. Nuevas Ideas en Informática Educativa TISE. 
Peletti, R. B. (2015). Micro dentista: Um jogo digital aplicado a saúde bucal. XIV Simpósio Brasileiro de Jogos e Entretenimento Digital, Teresina. Brasil, pages 304312.

Rocha, P., Nery Filho, J., and Alves, L. (2014). Jogos digitais e reabilitação neuropsicológica: delineando novas mídias. Anais do Seminário Tecnologias Aplicadas a Educação e Saúde, 1(1).

Rohde, L. A. P., Miguel Filho, E. C., Benetti, L. P. d. C., Gallois, C. B., and Kieling, C. C. (2004). Transtorno de déficit de atenção/hiperatividade na infância e na adolescência: considerações clínicas e terapêuticas. Revista de psiquiatria clínica. São Paulo. Vol. 31, n. 3 (2004), p. 124-131.

Silva, E. T., Ianaguivara, E. S., Gordinho, T. L., Martucci, H., Scardovelli, T. A., Bochi, S. R. M. S., and Silva, A. P. (2016). Jogo computadorizado para identificar características de falta de atenção e hiperatividade. XXV Congresso Brasileiro de Engenharia Biomédica - CBEB 2016.

Siqueira, C. M. and Gurgel-Giannetti, J. (2011). Mau desempenho escolar: uma visão atual. Revista da Associação Médica Brasileira, 57(1):78-87.

Wrońska, N., Garcia-Zapirain, B., and Mendez-Zorrilla, A. (2015). An ipad-based tool for improving the skills of children with attention deficit disorder. International journal of environmental research and public health, 12(6):6261-6280. 